\title{
El médico frente a la muerte.
}

The doctor facing death.

\section{Rosa Gómez Esteban a .}

${ }^{a}$ Psiquiatra Adjunto. Servicios de Salud Mental de Alcorcón, Madrid, España.

Correspondencia: Rosa Gómez Esteban (rosagomezesteban@gmail.com)

Recibido: 23/06/2011; aceptado: 01/08/2011

RESUMEN: se plantean los cambios en relación a la muerte a lo largo de la historia. Se recoge el discurso más significativo del proceso de un grupo de médicos cuya tarea era trabajar sobre la relación médicopaciente, analizando los emergentes surgidos en relación a la problemática de la muerte. Se evidencian en los médicos mecanismos defensivos de negación muy intensos frente a la muerte de los pacientes, mayores cuanto más compleja es su propia vinculación con la muerte. Se trabajan estas ansiedades y mecanismos defensivos en grupo operativo observando que es un instrumento de gran utilidad para la elaboración de estas dificultades de los médicos frente a la problemática de la muerte de los pacientes.

PALABRAS CLAVE: muerte, emergentes, mecanismos defensivos de negación, grupo operativo.
ABSTRACT: changes arise in relation to death throughout history. Collect the most significant speech of the process of a group of doctors whose task was to work on the doctor-patient relationship, analyzing the emerging arising in relation to the issue of death. Are evident in the medical defense mechanisms of denial intense face of death of patients over the more complex relationship with his own death. We work on these anxieties and defense mechanisms in noting that the task force is a useful tool for preparing for difficult medical problems facing the death of patients.

KEY WORDS:death, emerging defense mechanisms of denial, the task force.

\footnotetext{
"El hecho moderno es que ya no creemos en este mundo. Ni siquiera creemos en los acontecimientos que nos suceden, el amor y la muerte, como si sólo nos concernieran a medias..." (1)

"La vida de los médicos está llena de las angustias y emociones que rodean el tema de la muerte" (2)
}

\section{I.- Introducción}

La muerte siempre ha sido un acontecimiento complejo, pero las sociedades tradicionales atendieron y escucharon al moribundo hasta el último suspiro. La problemática de la muerte en la Edad Media es un asunto público y colectivo, se asume con naturalidad, como muestran algunas obras de la literatura que describen el correcto morir. En esa época, el médico tiene la obligación de informar al moribundo, en "El Quijote”, le aconseja: “cuide de la salud de su alma porque la de su 
cuerpo corre peligro". Aries (3) (4), en su libro "El médico ante la muerte" señala que el hombre de otro tiempo hacía caso de la muerte, era un cosa seria, grave y terrible, pero no tanto como para apartarla, huir y hacer como si no existiera.

A inicios del s. XIX surge el temor a la muerte, correlativo a la privación que se hace del derecho del hombre a saber que se va a morir. El médico, según un diccionario de ciencias médicas de la época, si no puede salvar a un enfermo debe evitar encontrarse a su lado en el último suspiro. A mediados de siglo es un deber de la sociedad mantener al moribundo en la ignorancia de su estado y, a finales del mismo, se modifica el primer período del morir, el de la enfermedad grave, circunstancias magistralmente reflejadas en la novela de Tolstoi, Iván Ilich (5).

A principios del s. XX, después de la primera guerra mundial, se prohíbe el duelo, y, después de la segunda gran guerra, se llega a la medicalización completa, se pierde lo único que se mantenía, el momento de la muerte con la revisión de la vida y la despedida. La sociedad ya no ayuda a morir a los enfermos, aunque se les alargue la vida gracias a los avances de la medicina; no se muere en casa y se generaliza la incineración como método para eliminar los muertos con discreción (6). Se acepta la prohibición de la muerte, no se habla de ella y el duelo de los allegados ha de realizarse en silencio, el hombre común y los especialistas se sustraen a esta reflexión. La muerte se convierte en un tabú social, en una cuestión individual y privada, se pasa de la muerte pública a la muerte oculta. Se impone la idea, aberrante para el hombre medieval, que es mejor no darse cuenta de que uno se está muriendo.

Aries precisa que de la muerte consciente, ritualizada y pública del medievo se pasa a la muerte temida, que desemboca en la muerte medicalizada, solitaria y vergonzante de hoy en día. Los enfermos en los hospitales no pueden despertar en los médicos y enfermeras la insoportable emoción de la muerte, son apreciados en la medida en que hacen olvidar al entorno médico que van a morir. Se transita desde una visión del moribundo que conserva su valor hasta el final, hasta la percepción actual en la que no tiene ningún valor social. La anulación se efectúa a pesar del deseo de los allegados de conservar su memoria, se niega el duelo a los supervivientes y esta supresión del duelo es una coacción de la sociedad, una manera de rechazar la presencia de la muerte, de excluirla. El duelo se reprime, aunque ello tenga importantes consecuencias para el sujeto.

Es preciso esperar al último tercio del s. XX para que una médica, KublerRoss (7), en su libro "La muerte y los moribundos", aborde la dignidad del moribundo y exija reconocerla como un acontecimiento esencial. En el siglo actual, la demanda social de ayudar a una muerte digna a los pacientes terminales, ha permitido en algunos países (Holanda, Bélgica y Luxemburgo), que el médico facilite una muerte tranquila, si es solicitada por un paciente terminal. En nuestro país, se dan actitudes opuestas, la negación y reducción de la muerte a un hecho biológico 
ORIGINALES Y REVISIONES

se nos ha mostrado en toda su dureza en el conflicto acaecido en el hospital "Severo Ochoa" de Leganés, Madrid, en la denuncia de la Consejería de Salud al Dr. Montes y su equipo.

Antes de centrarme en la problemática del médico y la muerte, deseo incluir las reflexiones acerca de la muerte de dos filósofos, muy distanciados en el tiempo y con dos posturas diferenciadas, Epicuro y Blanchot. Epicuro, en la Epístola a Meneceo, expresa que "el peor de los males, la muerte, no significa nada para nosotros, porque mientras vivimos no existe, y cuando está presente, nosotros no existimos. Añade que la mayoría de la gente, unas veces, rehúye la muerte viéndola como el mayor de los males y, otras, la invoca para remedio de las desgracias de esta vida. Y que el sabio, ni desea la vida, ni rehúye dejarla, porque para él el vivir no es un mal, ni considera que lo sea la muerte. Y así como entre los alimentos no escoge los más abundantes, sino los más agradables, disfruta no del tiempo más largo, sino del más intenso placer" (8).

Desde otra posición, Blanchot analiza la obra de Rilke y plantea que el hombre de antes sabía que la vida contenía la muerte y deseaba que no fuera un accidente, al interrogarse acerca del ser de la muerte, reflexiona que no es sólo una realidad biológica y que no ha de estar en el momento último, sino en la profundidad de la vida y formar parte de la existencia. La negación de la la muerte supone negar los aspectos graves y difíciles de la vida, y si no se aceptan los males de la vida, no se vive, por ello insiste en que la muerte es un más allá que se tiene que aprender, reconocer y acoger". La idea de que la muerte está en el corazón de las cosas, también se encuentra en la obra de otros dos filósofos del s. XX, Sartre y Camus, quienes describen la soledad, la incomunicación del hombre en la multitud y el sentimiento melancólico de la brevedad de la vida.

Las reflexiones posteriores están hechas a partir de la coordinación de un grupo de médicos, cuya tarea era trabajar sobre la Relación Médico - Paciente. Este grupo tenía un encuadre de frecuencia quincenal y hora y media de duración, a lo largo de seis meses. Fruto de esta experiencia y de la tesis doctoral sobre "las ansiedades del médico en la relación médico -paciente" es la publicación personal del libro "El médico como persona en la relación médico-paciente" (Gómez Esteban, 2002), (9), en el que se concluye que las ansiedades más intensas del médico derivan de la relación con el paciente. La relación insatisfactoria con éste y el sufrimiento derivado por la enfermedad, son las variables más significativas, que encuentran su máxima expresión en los enfermos graves y, sobre todo, terminales. La confrontación con la muerte, los moribundos y el duelo es una realidad cotidiana para los médicos en su práctica clínica. La muerte es uno de los problemas esenciales del hombre, frente a la que se presenta un intenso temor y los médicos como hombres que son, también sienten estos temores, por ello no pueden enfrentarla con serenidad, de acuerdo con Mc Cue (10) y Mingote Adán (11). A pesar de 
la importancia de estos aspectos en su quehacer clínico, esta problemática tan compleja no es abordada durante la formación, ni en la carrera de medicina ni durante los estudios de la especialidad. Las carencias en la formación se evidencian cuando el médico tiene que enfrentarse en su quehacer a estas circunstancias humanas, haciéndolas más dolorosas y angustiosas. La muerte del paciente está muy presente en el quehacer de los profesionales y les genera sentimientos muy diversos, entre ellos, impotencia y culpa, que pueden ser reprimidos, hacerse inconscientes y manifestarse a través de síntomas somáticos o psíquicos. Es un acontecimiento de su quehacer que viven con exigencia y temor, sobre todo, si la implicación ha sido excesiva o ha habido una mala praxis. Esta condición humana, real o imaginaria, en la que el médico se implica personal y profesionalmente, si no es reconocida y aceptada, hace que la muerte sea uno de los acontecimientos de mayor ansiedad de su quehacer clínico.

Nuestra tarea en los grupos fue hacer posible el intercambio de palabras y vivencias para que pudieran elaborar las dificultades frente a la muerte de los pacientes y dar a ésta su lugar en la práctica clínica. Las ansiedades, sentimientos y vivencias alrededor de la muerte se trabajaron fundamentalmente a lo largo de tres sesiones grupales hacia la mitad del proceso grupal y fueron tan significativas que motivaron, por primera vez, la ausencia de más de la mitad del grupo. El esquema referencial teórico que se utilizó es la metodología psicoanalítica de orientación lacaniana, que nos da una teoría del sujeto del inconsciente $\mathrm{y}$, para lo grupal, la teoría y técnica de grupo operativo, que incluye la metodología psicoanalítica, y es central para el análisis del discurso grupal. La articulación entre lo singular, grupal y social se muestra a través de los emergentes que, aunque manifestados por el sujeto, representan el discurso y acontecer grupal, por ello se han priorizado en el análisis de esta reflexión. Estos emergentes, interrelación entre la singularidad, que es lo diferente de cada sujeto, y lo acontecido en el grupo, permiten abordar las dificultades de la relación médico-paciente, que son mayores cuando la patología de éste es grave y terminal. El trabajo sobre la relación médico -paciente, sobre las ansiedades que se generan en el quehacer clínico es fundamental porque previene patología somática y de salud mental en el médico. En esta reflexión me centraré en tres aspectos de la problemática del morir: la información del médico al paciente, los aspectos psicológicos de la relación médico-paciente y la dinámica del proceso grupal, señalando algunos cambios que se producen a lo largo del mismo. 
ORIGINALES Y REVISIONES

\section{II.- Interrogantes del médico, informar: a quién, qué, cómo y cuando}

La información en las enfermedades graves o terminales siempre ha planteado un dilema ético al médico, debido a la actitud social de ocultación de la muerte. La institución sanitaria y los médicos dentro de ella han defendido la necesidad de ocultar al paciente todo lo referente a su enfermedad, en la idea de que no actuar de este modo produce un estado de desesperanza en el enfermo que repercute negativamente en su recuperación. La tendencia al encubrimiento se mantiene en la cultura médica hasta avanzado el siglo XX, pero a partir de 1977 se invierte al aprobarse en EE.UU. la "Ley del Derecho a la Información" que obliga al personal sanitario a dar el diagnóstico al enfermo. Este cambio legal permite que los profesionales sanitarios proporcionen información a sus pacientes, pero, con el paso del tiempo, surge un fenómeno paradójico, los pacientes empiezan a manifestar su deseo de no ser informados.

Aunque la ocultación de la verdad del diagnóstico y pronóstico presuponía desconfianza del médico en la madurez de sus enfermos para afrontar la enfermedad (12), la transmisión de la verdad sobre la enfermedad y el pronóstico exige un conocimiento de los pacientes y una formación adecuada de los profesionales. La formación que se propone desde nuestro esquema referencial psicoanalítico y grupal ha de integrar los aspectos técnicos y afectivos, es decir, ha de integrar la información acerca de la enfermedad y la reflexión sobre las emociones que se generan en el desarrollo de estos procesos. Es una formación que prioriza los aspectos emocionales del paciente, del médico y de los familiares, y tiene como objetivo dotar de instrumentos al médico para que pueda apoyar a los protagonistas del proceso.

Uno de los diagnósticos que mayor angustia moviliza es el cáncer, a pesar de que en la actualidad un alto porcentaje de los pacientes se curen (13). Los médicos con frecuencia ocultan el diagnóstico, aún en circunstancias en las que el enfermo les haya pedido la verdad, cuidar estos aspectos es fundamental porque, como hemos señalado, el sufrimiento del paciente deriva de la relación con el médico (14), (15), (16). El interrogante para el profesional es conocer a quién informar, ya que con frecuencia tiene dificultades para conocer si quiere o no saberlo. Esta problemática es el emergente central de las primeras sesiones del grupo, los profesionales refieren actitudes contradictorias derivadas de la negación y ambivalencia del paciente y la familia, se manifiesta asombro e incluso cierta perplejidad cuanto mayor es la actitud negadora del paciente, sobre todo, cuando éste previamente ha solicitado información.

En el grupo pueden tomar conciencia de la ambivalencia del enfermo en lo que se refiere a conocer su pronóstico, se sorprenden ante sus conductas contradictorias y su deseo de ser engañados. Hacerse conscientes de este deseo contradictorio de 
saber y no saber del paciente, les permite reflexionar sobre su propia ambivalencia y pensar que estos sentimientos contradictorios frente a la muerte están presentes en ambos. La aceptación de estos sentimientos compartidos, les permite pensar en la importancia de la personalidad del paciente y de la investigación de su demanda, para poder transmitir la información adecuada de acuerdo a sus necesidades y no por imperativo legal. La comprensión de que los temores frente a la muerte están en el origen de esta ambivalencia de sentimientos, les permite centrarse en la demanda del paciente y familiares que, en ocasiones, son contradictorias y ponen en un difícil situación al médico.

Trabajar en estos aspectos les permite descubrir dos modalidades de enfermos: los que desean conocer y evitar la conspiración del silencio y, los que no desean saber, aunque afronten la enfermedad con negación, ansiedad y depresión, desconociendo que estos afectos empeoran la evolución y el tratamiento. La detección de las demandas contradictorias del paciente, les permite reflexionar que si la información es adecuada, el efecto es beneficioso, ya que disminuye la angustia del paciente, pero esta información ha de ser pertinente y ofrecerse en el momento adecuado.

El médico puede reconocer que estos mecanismos son defensas frente a la ansiedad que les genera la muerte, y esta reflexión permite que se manifiesten los siguientes interrogantes: ¿Qué informar, cómo y a quién? A estas preguntas respondemos con Ibáñez (17), a quien pida, busca y quiera información, pues quien nada pregunta, nada quiere saber; teniendo en cuenta que el límite de la información es el deseo de no saber del paciente, como señalan Caretti (18) y De Ángel Martín (19).

La información, por tanto, a un enfermo de cáncer o de cualquier patología que comprometa la vida, ha de darse paso a paso, teniendo en cuenta las distintas etapas que atraviesa el paciente y no hacerlo abruptamente como hemos observado en algunas patologías como el sida. Informar por informar, satisface los requisitos legales que la sociedad impone, pero no cumple los principios éticos que toda actividad terapéutica demanda, de acuerdo con García Conde (20). La información a los pacientes con cáncer favorece la adaptación psicológica, el nivel previo de funcionamiento y la disminución de la sintomatología psicopatológica que aparece al inicio de la enfermedad. Se ha constatado que los pacientes informados, con el tiempo aprenden a convivir con su enfermedad, mientras que los que no conocen su pronóstico, lo sospechan y muestran más ansiedad. Por otro lado, hay que tener en cuenta que las significaciones individuales y sociales de la enfermedad influyen en la adaptación a la enfermedad, los estudios muestran que los sentimientos y vivencias del paciente dependen de estas creencias (21-22).

Es necesario hablar con los pacientes acerca de la situación en la que se encuentran para que puedan enfrentarse mejor a la enfermedad y prepararse para la muerte (23). Freud, a principios del siglo XX, expresó este deseo cuando llegara el 
ORIGINALES Y REVISIONES

momento de su muerte, demandaba que se le tratara con respeto y se le advirtiera para estar preparado, quería tener información sobre cualquier fase de su enfermedad y pedía que se le permitiera morir con dignidad (24).

\section{III.- Negación de la muerte y relación médico-paciente}

En la relación médico-paciente, las ansiedades más intensas en el enfermo, se derivan del pronóstico de su enfermedad, y en el médico, si se dan carencias personales para enfrentar el sufrimiento y la muerte del paciente, o cuando existe desconocimiento de algún aspecto de la enfermedad. En esta relación, a la ansiedad del enfermo y familiares, el médico responde con la suya propia, generándose en algunas circunstancias una cadena de acontecimientos que son el origen del engaño, la incomunicación y el aislamiento del enfermo, en estas circunstancias, el propio médico para disminuir su ansiedad evita el contacto directo con el enfermo. En este círculo de ocultamientos e incomunicación, el paciente aprende a no preguntar por temor a la respuesta y para no poner en apuros al médico, sobre todo, si observa que éste elude la cuestión, tampoco lo hace con los familiares para no hacerles sentirse culpables. De este modo, el paciente empieza a vivir en solitario lo más esencial de su vida, la propia muerte.

Estas vivencias las vemos reflejadas magistralmente en la obra de Tolstoi, "La muerte de Iván Ilich" es una mirada directa a ese vacío que tanta angustia nos produce, la muerte. Iván Ilich, después de haber llegado a la cima de su carrera profesional, se enferma, primero hace una negación de su enfermedad, después se siente enfermo, se siente morir y se plantea el por qué de esa muerte y de esa soledad que no le deja vivir, vive en soledad su muerte porque el médico y los familiares más allegados hacen una negación de la misma. Iván, después de la negación inicial, comprende que el problema no es qué órgano está enfermo, sino que se trata de su vida y su muerte. Su principal tormento es la mentira admitida por todos de que él sólo está enfermo y esta mentira se le hace muy penosa en vísperas de su muerte. Manifestar su agresividad le permite pedir que se le deje morir en paz, expresar su sufrimiento, aceptar la muerte y despedirse de sus seres queridos.

En la relación médico-paciente, en los momentos cercanos a la muerte, es habitual que surjan malentendidos e incomunicación, pero como nos recuerda Tolstoi, el problema se produce cuando las dificultades se incrementan y se produce el aislamiento del enfermo, quien vivirá en soledad este momento crucial de su vida. Ya Freud nos señalaba que el temor a la muerte de los pacientes era uno de los aspectos más difíciles de la medicina y que el médico no debía engañar a los enfermos. Pero, uno de los factores que contribuye al engaño y al aislamiento del 
paciente es la problemática del médico respecto a la muerte, cuando éste proyecta sobre el enfermo sus temores frente a la misma. Tener en cuenta estas cuestiones es fundamental porque cuando los temores y mecanismos defensivos en ambos son intensos se pueden desencadenar fenómenos de inhibición y/o crisis de angustia en el paciente, pero también en el médico, haciendo más difícil este proceso. La inclusión de los temores, ansiedades y mecanismos defensivos del médico nos permite comprender la dinámica de la relación y entender las dificultades de algunos pacientes en el trabajo de elaboración del final de su vida.

En el proceso grupal el médico puede descubrir los mecanismos de negación que surgen frente a la muerte, tanto los del paciente como los propios. La reflexión se puede seguir a través de los interrogantes que se plantean a lo largo del desarrollo grupal. Al inicio, cuando se preguntan: “ ¿El paciente puede querer no saber?”, lo que les lleva a descubrir el "deseo de no saber del paciente". Y del reconocimiento de este deseo del enfermo a la pregunta acerca del propio y al encuentro con el "deseo de no saber del médico". De estos mecanismos defensivos de negación, tanto del paciente como del médico, surgen numerosos emergentes, que muestran la necesidad del hombre de huir frente a la muerte. El hombre huye de la muerte, es una realidad dolorosa, para el enfermo significa la pérdida de su vida y para el médico encontrarse con sus limitaciones, con su ignorancia.

Las complejas negaciones, conscientes e inconscientes, y la huida del profesional frente a la muerte del paciente, nos acercan a Kübler-Ross, cuando señala que la pregunta no es si el médico debe informar o no, sino cómo compartir este acontecimiento con el paciente, ya que todos sus enfermos moribundos conocían su situación aunque no se les hubiera informado, lo único que pedían era que el médico lo hiciera de una manera aceptable. En su libro "Sobre la muerte y los moribundos" hace numerosas referencias a pacientes que no pudieron compartir estos momentos fundamentales de su vida por la intensa ansiedad de los profesionales. Si para el médico la muerte es tabú y terrible, como algunos médicos reconocían en el grupo, el profesional no puede ayudar al paciente a afrontar la muerte con serenidad. De acuerdo con esta autora, la capacidad de negación del paciente es directamente proporcional a la del médico, si éste se niega esta verdad a sí mismo, lo niega en sus pacientes. Sin embargo, cuando en el profesional disminuyen los temores y los mecanismos de negación, lo pueden hablar en el grupo y con sus pacientes, se produce un cambio que se manifiesta en el reconocimiento de que éstos son capaces de afrontar su muerte.

Los temores y ansiedades del médico son mayores cuando se teme que la información pueda desencadenar el suicidio del paciente. Esta situación se produce con mayor frecuencia cuando la enfermedad orgánica se asocia a depresión grave, pero si bien es cierto que estas situaciones requieren extremar el cuidado en la información que se le ofrece al paciente, en ocasiones, es un temor sobrevalorado 
ORIGINALES Y REVISIONES

para no afrontar las dificultades inherentes a estos procesos. El profesional no pregunta al paciente porque teme que su intervención pueda precipitarlo, sin embargo, es importante que el médico investigue acerca de estas ideas suicidas cuando lo sospeche, ya que estas preguntas son la posibilidad de poner los medios para su prevención. Es necesario revisar nuestras concepciones acerca del suicidio, ya que los temores y prejuicios no permiten preguntar por estos deseos de muerte y el problema es que cuando se adoptan estas actitudes moralistas se da la espalda a esta dolorosa realidad y se abandona al paciente, lo que sí puede precipitar el acto del enfermo.

Los prejuicios acerca del suicidio los podemos observar en la muerte, por algunos cuestionada, de Deleuze. Las duras críticas ocultan la realidad de que el fil para Deleuzente, ios las podemos observar en uso en la informaciósofo padecía una fibrosis quística pulmonar y que estuvo cerca de 30 años enfermo antes de su muerte, los últimos cinco conectados a una máquina, sin apenas poder hablar y escribir. Sin embargo, Rolnik (25) hace una interpretación muy distinta de este acontecimiento, refiere que el filósofo quiso seguir viviendo con graves restricciones y dificultades, hasta que todo se volvió imposible y escogió hacer lo que trató de hacer siempre en su vida y defendió en su obra, afirmar la vida hasta el extremo de su fin y enfrentar la diferencia más insoportable, la que requiere más coraje, el enfrentamiento con la muerte.

La negación de la muerte en la práctica médica, lleva a una falta de percepción de la situación y, por tanto, a una falta de intervención, de este modo, no se aborda la atención psicológica de la persona que va a morir. La medicina tecnificada actual olvida que los individuos han de decidir y colaborar de manera responsable en la asistencia a su propia muerte. El olvido, como hemos señalado, es efecto del contexto socio - familiar que oculta la muerte desde la infancia y la considera un hecho del que no se debe hablar. Esta ocultación se intensifica por la hegemonía del modelo biológico, que determina que en la medicina el médico tenga sólo en cuenta los aspectos biológicos de la enfermedad.

En la medicina actual se reconoce y asiste a la muerte corporal pero no al hecho psicológico y social (26). Esta concepción, hemos señalado que es producto de una cadena de negaciones que se transmiten a través de las generaciones, en unas condiciones histórico y sociales que determinan creencias y modalidades de comportamiento en la institución médica, familiar y en el propio enfermo. El paciente niega la gravedad de su enfermedad, y al hacerlo, niega la posibilidad de su muerte, con lo que ni se plantea solicitar ayuda al otro. La muerte es negada en la Medicina y en la extensa formación de los profesionales, aunque paradójicamente el aprendizaje se inicie en el contacto con el cadáver. Estas carencias en la formación influyen en la práctica clínica y en la relación médico-paciente y también en los mecanismos defensivos inconscientes del profesional frente a la muerte, de los 
que hemos señalado la negación, pero que también incluyen otros, como la evitación y proyección.

En casos extremos, el comportamiento del médico puede oscilar entre el abandono o la identificación masiva con el enfermo y la familia, haciendo inviable su función terapéutica. Estas actitudes extremas se muestran en estos dos emergentes grupales: "Quieres desaparecer del mapa" vs "Hay que preservarse, si no te mueres con cada uno un poco". Mostrar esta necesidad de huida o la identificación masiva en los momentos cercanos a la muerte de sus pacientes, les permite expresar su necesidad de ayuda para establecer una distancia terapéutica con el paciente y su familia ("Los pacientes necesitan apoyo, pero también tú para poner límites, si no la angustia de los otros te invade"). Cuando se dan masivas identificaciones con el paciente o la familia pueden surgir conductas paradójicas en el médico: "No puedes llorar por lo que le pasa, para eso está la familia, prefiero hacer bromas con el paciente y que no piense en lo que tiene". Estos sentimientos son más intensos si la muerte ha cuestionado la omnipotencia del profesional o si la significación de la muerte del paciente supone un fracaso personal. En estos casos se puede oscilar entre la omnipotencia y la impotencia, entre estos sentimientos y la culpa. El grupo tiene gran interés porque permite percibir estos afectos, disminuir la angustia y continuar más serenos con su proyecto como profesionales.

Para entender los mecanismos defensivos del médico y del paciente hacia la muerte nos resulta de gran interés las aportaciones psicoanalíticas de Freud (27), en su obra "Consideraciones de actualidad sobre la guerra y la muerte", quien plantea que el hombre muestra una inclinación a prescindir de la muerte y a eliminarla de la vida. Considera que la muerte propia es inimaginable, que en el fondo nadie cree en su propia muerte ya que en lo inconsciente se está convencido de la inmortalidad y el hombre va aceptándola a través de la muerte de los otros significativos para él o de las pérdidas que se viven a lo largo de la vida.

Freud afirma que el miedo a la muerte que domina al hombre es más frecuente de lo que se reconoce y procede casi siempre del sentimiento de culpabilidad y que el hombre puede aceptar la muerte si se trata de un extraño o de un enemigo, pero no cuando se trata de sus personas amadas. Los sentimientos de culpa provienen de los pensamientos o sentimientos respecto a los seres queridos que se han reprimido en el inconsciente. Nuestro pensamiento puede asesinar por lo que aparentemente son pequeñeces, porque cualquier daño realizado a nuestro omnipotente y despótico "yo" se considera en el fondo un crimen. El inconsciente puede alojar violentos deseos de muerte frente a aquellos que nos han ofendido o perjudicado.

Freud descubre en la clínica ese deseo de matar a la persona amada, ya que junto al cariño se encuentra una hostilidad latente que puede estimular el deseo inconsciente de muerte. Freud nos muestra la ambivalencia afectiva, a través de la experiencia analítica, y deduce la importancia de los deseos inconscientes de muer- 
ORIGINALES Y REVISIONES

te en sujetos que presentan autorreproches y preocupación exagerada por el bien de los familiares, después de haber sufrido la muerte de la persona amada. Esta ambivalencia de sentimientos es más marcada en algunas patologías que observamos en la clínica, por ejemplo, en la neurosis obsesiva y en la melancolía.

Como plantea Freud, la muerte es más traumática en la medida en que se conecta con deseos de muerte que inconscientemente se han tenido respecto a las personas significativas. Es decir, cuanto más el médico haya reprimido los sentimientos y pensamientos de muerte hacia los otros, más mecanismos defensivos tendrá que poner en marcha para enfrentar/evitar la muerte. Los médicos que son más negadores con sus sentimientos y afectos, más lo son con los de los otros y, más aún, si estos pensamientos tienen relación con sentimientos de hostilidad y de odio. Cuanto más intensos son los deseos de muerte inconsciente hacia los otros, mayor represión, negación y mayores sentimientos conflictivos, entre ellos la culpa, impotencia y cobardía, que van a dificultar acercarse a la problemática de la muerte a estos profesionales.

IV.- Proceso grupal: de no “querer saber” a ¿cómo acompañar al paciente moribundo?

El desarrollo del grupo se centró alrededor de tres interrogantes, el primero referido a la información, “Debe informarse, quién, qué, cómo y cuando?” y el segundo, al deseo de no saber del enfermo y del médico ( El paciente puede querer no saber?, “a mí, si me conocieran, no me lo dirían”), ambos ya señalados. El tercer interrogante se refiere a la intervención del profesional: ¿Qué ha de hacer el médico ante la muerte del paciente?

Aunque el médico tenga clara la obligación de informar al paciente y familiares, con frecuencia se siente inhibido y bloqueado en su tarea clínica porque no sabe qué hacer ante la muerte de sus pacientes. El imperativo ético de informar puede significar mayor exigencia superyoica e inhibición, como observamos en el bloqueo relatado con gran emoción por una de las médicas del grupo: "Me preguntó cogiéndome del cuello, ¿es un cáncer?, no fui capaz de decírselo, me había preparado y no pude decirle la verdad, después ya no habló más. Me acobardé, me lo preguntó entre gritos y lloros, me pareció que quería que le dijera que no, se murió sospechando y sola, os tenía que haber pedido ayuda".

Si la inhibición del médico se intensifica, el profesional puede sentirse incapaz de dar el pésame a la familia ("Yo sonrío y salgo lo más rápido posible de la habitación; si muere, el pésame no se lo doy a la familia, no puedo"). Frente a esta reflexión que conmovió al grupo, como otras que se produjeron, señalo las palabras 
de Fernández, (28), "lo que nos define como humanos no es el morir, pero sí saber que forma parte de nuestra condición... y, sin embargo, vivir con la mayor intensidad y esmero. Conocer el poder de la muerte y no dejar que él destruya la vida por anticipado, resistirse a la muerte con todas las ganas de la vida".

El tercer interrogante acerca de la intervención del médico surge cuando disminuyen los mecanismos de negación y se puede hablar de los afectos y sentimientos, entre ellos, la angustia, la ansiedad, la depresión y la culpa. El médico acepta que puede informar de los procesos biológicos, del diagnóstico, pronóstico y tratamiento, pero tener dificultades para abordar los problemas existenciales que se plantea el paciente en estas circunstancias vitales, reconociendo, de este modo, su desconocimiento y cómo influye en las vivencias del paciente. El dispositivo grupal ofrece a los médicos la posibilidad de mostrar sus limitaciones y contradicciones y preguntarse acerca de su función en estas circunstancias humanas. En el grupo los médicos pudieron hacer un cambio en su posición, desde el rol técnico que niega la importancia de su función en el fin de la vida de sus pacientes, al reconocimiento de su impotencia e ignorancia. Desde el rol de expendedor de medicamentos ("Somos los que hacemos las recetas y calmamos el dolor, nada más"), a la pregunta sobre su quehacer con el paciente moribundo (¿Qué tenía que haber hecho?, no lo sé, ella murió mal).

Pueden reflexionar que no se trata de la información de lo biológico, que es un saber conocido por el médico, sino del trabajo de lo psicológico, con frecuencia desconocido y temido, en el que el médico teme dañar al paciente y viceversa. El médico acepta que no suele estar preparado para las muertes simbólicas que el paciente ha de elaborar hasta que se produzca el óbito, que la dificultad es el drama existencial del paciente y de lo que se trata es de prepararse para lo humano que se moviliza frente a la muerte. El reconocimiento de estas carencias les permite un cambio, solicitar ayuda para afrontar las dificultades del quehacer clínico y considerar que éste incluye, saber contener y acompañar al paciente.

La cuestión, por tanto, es cómo se acompaña a un moribundo, cómo se le ayuda a que pase el tránsito de la vida a la no existencia, cómo se le acompaña conociendo las dificultades del hombre frente a la muerte. Kübler-Ross anima a que en este difícil trance, el médico escuche con serenidad, la rabia, el llanto, el dolor y los temores y fantasías de sus pacientes. Propone ayudarles a separarse de sus relaciones significativas y así aceptar su muerte, apo poaque pueda dar aceptar que una pa: "yar a la familia para que comprendan que la muerte puede ser un gran alivio, y que es más fácil si se les ayuda a desligarse lentamente de las relaciones importantes de su vida. Para conseguir este objetivo, como dice la autora, el médico debe respetar los mecanismos defensivos del paciente y ayudarle cuando esté en disposición de aceptar la ayuda. Y, cuando llegue el momento final, estar presente en silencio para confirmar que se está disponible hasta el final, ya que para el pa- 
ORIGINALES Y REVISIONES

ciente es muy reconfortante saber que no se le olvida, aunque no se pueda hacer nada más por él. También aconseja apoyar a la familia para que de expresión a sus sentimientos antes de que se produzca la muerte, ya que ello posibilita su elaboración y previene los duelos patológicos causantes de tantas enfermedades mentales y somáticas posteriormente.

La elaboración de los afectos y sentimientos frente a la muerte de sus pacientes es una tarea más necesaria si el profesional tiene rasgos obsesivos o melancólicos, si no se realiza este trabajo es mayor el riesgo de identificaciones patológicas que tanto daño hacen al paciente y familiares. En estos casos, el médico puede llegar a una situación de confusión, experimentar intensa ansiedad y defensivamente responder con actitudes de frialdad, huida y abandono del paciente. Si esta ansiedad no es elaborada, el profesional no toma la distancia terapéutica adecuada y, por tanto, no puede informar, acompañar y apoyar al paciente y a la familia en tan dolorosas circunstancias. Insistimos en la necesaria elaboración de esta problemática, porque su falta puede desencadenar actitudes y comportamientos patológicos incomprensibles para los pacientes, en un momento en que su deseo fundamental es sentirse acompañados. En estas circunstancias el sufrimiento del paciente se incrementa ante su temor a la incomunicación y al abandono, al saber que va a morir y no puede esperar nada del médico que le atiende.

Haciendo una evaluación del trabajo grupal, consideramos que el grupo se ha mostrado como un instrumento de gran interés, los médicos pudieron transitar desde la necesidad de engaño del paciente a su propia necesidad de ser engañados, pudieron vivenciar el impacto que les produce la muerte y el sufrimiento del enfermo, como aquella médica que refería su imposibilidad de confirmar el diagnóstico que ya le había hecho la paciente, a pesar de haberse preparado para decirle la verdad. También pudieron reconocer sus mecanismos de identificación con el enfermo y disminuir aquellas identificaciones patológicas que no les permite escuchar y conocer sus necesidades, como aquel que decía que se moría con cada paciente. Por otro lado, los profesionales manifestaron sus sentimientos de cobardía y de impotencia, como aquel que defendía la postura de que no había qué decir la verdad al paciente, porque no se iba a curar y se le iba a dejar hecho polvo. También reconocen que sus limitaciones y "no saber que hacer" son la causa de ciertas conductas del médico que aumentan el sufrimiento del paciente y la familia, sobre todo, cuando el profesional se empeña en prolongar la vida al enfermo, en ir contra la muerte.

Esta reflexión acerca de sus límites les permitió disminuir su impotencia y pensar que, aunque no pudieran curar al paciente, si podían hablar con él o acompañarle mientras tuviera vida. El efecto de este diálogo con uno mismo y con los otros fue la disminución de los sentimientos de culpa y la toma de conciencia del sufrimiento del enfermo, del que referían un menor sufrimiento físico actual, pero más emocional. Los profesionales pudieron profundizar en las cuatro etapas que 
atraviesa el paciente en la aceptación de la muerte, descritas por Kübler-Ross: la primera, la negación y el aislamiento, la segunda, el resentimiento o la ira, la tercera, el pacto y la depresión y, la última, la aceptación.

Los médicos pudieron reconocer la necesidad de cuidarse para cuidar a los otros y plantearse la necesidad de buscar estrategias que no dañen la salud mental del paciente, de la familia y del propio médico. También de los efectos de estas carencias en la salud observaron que los mecanismos defensivos, si no eran intensos, eran necesarios para conseguir un vínculo más saludable. La necesidad de cuidarse les permite profundizar en la importancia de la función del médico como cuidador en estas difíciles circunstancias humanas y pensar que una de las tareas importantes es facilitar al paciente que acepte el final de su vida y pueda despedirse de sus seres queridos y amigos.

Insistimos en que la formación del médico, durante su etapa de residente, ha de incluir espacios de elaboración de las ansiedades frente a la muerte de sus pacientes, para que puedan contenerse las intensas ansiedades que se generan en estos procesos. Ya que, si el médico está preparado, podrá ayudar y acompañar al paciente y a la familia a enfrentarse mejor al final de la vida. Esta cuestión es central, ya que el acto médico, como hemos mostrado, está lleno de situaciones en las que la muerte está presente, aunque sea a nivel imaginario, también en aquellos momentos en los que ni médico ni paciente conocen la evolución de la enfermedad. Saber tratar estos aspectos es importante, ya que supone la incorporación de la muerte en la vida cotidiana del hombre, hecho fundamental para la existencia humana.

Las metodologías de grupo Balint o de grupo Operativo se han mostrado de gran utilidad para que los médicos elaboren o disminuyan sus ansiedades frente a la muerte de los pacientes. No se trata de crear una nueva especialidad, como dice Kübler-Ross, sino de formar a los profesionales sanitarios para que sepan enfrentarse a estas situaciones, una formación que tenga en cuenta las necesidades de los pacientes y profesionales. En estos espacios el médico puede aprender la importancia de la "escucha" del paciente y experimentar la necesidad de tener "otro" que te escuche en estas circunstancias humanas. Cuando el médico puede hablar de sus creencias, actitudes, sentimientos y vivencias hacia la enfermedad grave y la muerte, se disminuyen sus inhibiciones, bloqueos y resistencias en la relación médico-paciente.

Los profesionales que saben enfrentarse a la soledad, están más fortalecidos frente a los acontecimientos dolorosos de la vida, las separaciones y los duelos, como señala Hirigoyen (29), "afrontar la soledad consiste en mirar de frente el miedo a la muerte". Estos espacios grupales de intercambio permiten la integración de lo biológico y lo psicológico-social de sus enfermos, pero, han de tener un tiempo suficiente, para que el médico pueda reflexionar acerca de la relación humana con el paciente y aprender a "saber estar ahí con el enfermo", cuando no se sabe qué hacer ni qué decir, como dice Ingala (30). 
El médico ha de aprender a no hacer nada, que no significa dejar morir, sino permitirlo, tratar de evitar el sufrimiento a quienes van a morir y procurar ayudarles a vivir mejor, hasta que llegue la muerte. No encuentro mejores palabras, para terminar, que la pregunta que Freud nos plantea: “ ¿No sería mejor dar a la muerte, en la realidad y en nuestros pensamientos, el lugar que le corresponde y dejar volver a la superficie nuestra actitud inconsciente ante la muerte, que hasta ahora hemos reprimido tan cuidadosamente? Esta actitud ofrece la ventaja de tener más en cuenta la verdad y hacer más soportable la vida..., si quieres soportar la vida, prepárate para la muerte" (31).

\section{BIBLIOGRAFÍA:}

(1) Deleuze, G. , La imagen-tiempo. Estudios sobre cine 2, Barcelona, Paidós, 1987, p. 229.

(2) Álvarez L.F., La vida del médico, Barcelona, Ed. L.Caralt, 1949.

(3) Aries, PH., El médico ante la muerte ("Orígenes del gran miedo a la muerte"), Madrid, Ed. Taurus, 1983.

(4) Aries, PH., Historia de la muerte en occidente, desde la Edad Media hasta nuestros dn de Roland, a principios del siglo XI describe el correcto morir. tros dno mo a los sobrevivientes, por eso el duelo reprimido ías, Barcelona, El Acantilado, 2000.

(5) Tolstoi, L., La muerte de Ivan Ilich, Barcelona, Ed. Orbis, 1982.

(6) Buero, A., Pequeño ensayo sobre la muerte, Rev, Argentina de Cardiología, vol. 76, nº 5, sept-oct 2008.

(7) Kübler - Ross, E., Sobre la muerte y los moribundos, Barcelona, Grijalbo, 1994.

(8) Lledó, E., El epicureísmo, Madrid, Taurus Bolsillo, 1996.

(9) Gómez Esteban, R., El médico como persona en la relación médico-paciente”, Madrid, Ed. Fundamentos, 2002.

(10) Mc Cue, J.D., "The effects of stress on physicians and their medical practice", N. Engl. J. Med., 1982, 306: 458 - 463.

(11) Mingote Adán, J.C.; Pérez Corral, F., El estrés del médico, manual de autoayuda", Madrid, Ed. Díaz de Santos, 1999.

(12) Urraca Martínez, S., "La comunicación no verbal en la relación médico - enfermo", Jano, 1991, 953: 75-80.

(13) Thomsen, O.O.; Wulff, H.R.; Martín, A.; Singer, P.A., "What do gastroenterologists in Europe tell cancer patients?", Lancet, 1993, 8843: 473- 476.

(14) Gómez Esteban, R. Tesis doctoral sobre las ansiedades del médico en la relación médicopaciente, Universidad Autónoma de Madrid, Facultad de Medicina, 2000.

(15) Ruckdeschel, J.C.; Blanchard, C.G.; Albrech, T., Psychosocial oncology research. Where we have been, where we are going, and why we will not get there", Rev. Psic. Onc., 1994, 74: 1458 -1463.

(16) Yllá, L., "Lo inconsciente en las enfermedades terminales", en Psicología Médica, Ridruejo Alonso; Medina León; Rubio Sánchez; Madrid, Mc Graw - Hill Interamericana, 1997.

(17) Ibáñez, E., "Reflexiones acerca de las relaciones entre depresión y cáncer", Boletín de Psicología, 1984, 4: 99 - 117. 
(18) Caretti Ríos, J., "El problema del bien en la relación médico - paciente" en Medicina y Psicoanálisis, Madrid, Cepyp, Ed. Grafiprint, 1997.

(19) De Ángel Martín, L., "Información y verdad" en Medicina y Psicoanálisis, Madrid, Cepyp, Ed. Grafiprint, 1997.

(20) García Conde, J.; Pascual A., "La relación médico - enfermo en oncología" en Psicología y Medicina, Ed. Promolibro, Valencia, 1989.

(21) Ibáñez, E., "Los efectos de la información sobre los enfermos”, Jano, 1991, 953: 47-55.

(22) Durá, E.; Ibáñez, E., "La importancia de la congruencia informativa en el proceso de dar información al enfermo", Jano, 1991, 953: 57- 66.

(23) Nuland, S., Como Morimos, reflexiones sobre el último capítulo de la vida, Madrid, Alianza Ed., 1995.

(24) Schur, M., Sigmund Freud, enfermedad y muerte en su vida y en su obra, Barcelona, Ed. Paidós, 1972.

(25) Neto, L.; Gadelha, S., "Despedirse del Absoluto", Entrevista a Suely Rolnik, Nadie es deleuziano en "O Povo", Cuaderno Sábado, n 6, Fortaleza, 18 noviembre 1995.

(26) Santo Domingo, J., Psicosociología de la muerte, Madrid, Ed. Castellote, 1976.

(27) Freud, S., "Consideraciones de actualidad sobre la guerra y la muerte", en Obras Completas, 6: 2101-2118, Ed. Biblioteca Nueva, 1972.

(28) Fernández. E., "Extraña condición humana, reflexiones filosóficas", Madrid, Pub. uso interno Cepyp, 1999.

(29) Hirigoyen, M.F., Las nuevas soledades, el reto de las relaciones personales en el mundo de hoy, Barcelona, Paidós, 2008. 1997.

(30) Ingala, A., "Todo fracasa en el saber" en Medicina y Psicoanálisis, Cepyp, Ed. Grafiprint,

(31) Freud, S., ibídem. 